\title{
Pulmonal-arterielle Hypertonie und chronisch-thromboembolische pulmonale Hypertonie: Eine immunologische Perspektive
}

\author{
Thomas Koudstaal Karin A. Boomars Mirjam Kool \\ Department of Pulmonary Medicine, Erasmus MC, Rotterdam, Niederlande
}

\section{Schlüsselwörter}

Pulmonal-arterielle Hypertonie · Chronisch-

thromboembolische pulmonale Hypertonie · Entzündung und Immunität · Zirkulierende inflammatorische Biomarker .

Immunmodulatorische Therapie

\begin{abstract}
Zusammenfassung
Pulmonale Hypertonie (PH) ist eine extrem belastende fortschreitende Erkrankung, die durch erhöhte pulmonal-arterielle Drücke gekennzeichnet ist. Diese führen zu rechtsventrikulärer (RV) Insuffizienz (Rechtsherzinsuffizienz), Herzinsuffizienz und letztlich zum Tod. Je nach Ätiologie werden Patienten mit PH in die folgenden 5 Gruppen eingeteilt: (1) Pulmonal-arterielle Hypertonie (PAH), (2) PH bei Erkrankungen des linken Herzens, (3) PH bei Lungenerkrankungen und/oder Hypoxie, (4) PH aufgrund chronischer Thromboembolien (CTEPH) und (5) PH mit unklaren multifaktoriellen Mechanismen. Derzeit ist die Prognose für Patienten mit PAH bzw. CTEPH, selbst mit PAH-spezifischer Pharmakotherapie, weiterhin schlecht. Die durchschnittliche 5-Jahres-Überlebensrate beträgt für PAH 57\%-59\% und für inoperable CTEPH 53\%-69\%. Daher ist ein besserer Einblick in die Pathogenese von PAH und CTEPH dringend erforderlich, um neue Behandlungsstrategien entwickeln zu können. Neuere Studien zeigen sowohl bei PAH- als auch bei CTEPHPatienten ein erhöhtes Vorliegen und eine vermehrte Aktivierung angeborener und adaptiver Immunzellen. Darüber hinaus haben umfassende Forschungsarbeiten zu Biomarkern ergeben, dass
\end{abstract}

zahlreiche Entzündungs- und Immunmarker bei PAH- und CTEPHPatienten mit deren Hämodynamik und/oder Prognose korrelieren. Zunehmende Evidenzdaten über die pathologische Rolle von Immunzellen bei angeborener und adaptiver Immunität haben zu zahlreichen vielversprechenden präklinischen interventionellen Studien geführt. Diese ziehen ihrerseits innovative klinische Studien nach sich, die derzeit durchgeführt werden. Möglicherweise ist neben den aktuellen Behandlungen, die sich ausschließlich auf eine Vasodilatation stützen, eine Kombination immunmodulatorischer Therapien erforderlich, um eine wirksame Behandlung und Prävention gegen das Fortschreiten dieser Erkrankung zu etablieren. In dieser Übersichtsarbeit beschreiben wir die aktuellen Fortschritte bezüglich unserer Erkenntnisse über die Beteiligung der einzelnen Zelltypen des Immunsystems bei PH. Wir geben einen Überblick über den wachsenden Korpus an Evidenzdaten zu Entzündung und Immunität im Rahmen der Pathogenese von PH sowie über die Anwendung von inflammatorischen Biomarkern und immunmodulatorischen Therapien bei PAH und CTEPH.

(C) 2020 Die Autoren

Lizenznehmer MDPI, Basel, Schweiz karger@karger.com www.karger.com/kkp
This article is licensed under the Creative Commons Attribution 4.0 International License (CC BY) (http://www.karger.com/Services/Open AccessLicense). Usage, derivative works and distribution are permitted provided that proper credit is given to the au thor and the original publisher.

\footnotetext{
Thomas Koudstaal

Department of Pulmonary Medicine, Erasmus MC

Doctor Molenwaterplein 40
}

3015 GD Rotterdam, Niederlande

t.koudstaal.1@erasmusmc.nl 


\section{Einführung}

Pulmonale Hypertonie (PH) ist eine verheerende Erkrankung, die sich durch einen erhöhten pulmonalen Gefäßwiderstand und einen erhöhten pulmonal-arteriellen Druck auszeichnet. Laut der kürzlich aktualisierten Definition liegt PH bei einem erhöhten mittleren Pulmonalarteriendruck (PAP) in Ruhe über $20 \mathrm{mmHg}$ vor [1]. Dieser erhöhte pulmonal-arterielle Druck führt zu einer verstärkten rechtsventrikulären (RV) Hypertrophie, zu Herzinsuffizienz und schließlich zum Tod [2]. Ein strukturelles Remodeling des Gefäßsystems, das zu einer Verminderung des Gefäßdurchmessers führt, steht im Zusammenhang mit einem erhöhten pulmonalen Gefäßwiderstand und erhöhten pulmonalen Drücken. Der Schweregrad der PH wird nach der funktionellen Klassifikation (FC) der New York Heart Association (NYHA) angegeben und reicht von keinen Symptomen (Klasse I) bis schweren Symptomen in Ruhe (Klasse IV).

Basierend auf der zugrunde liegenden Ätiologie der PH werden gemäß der WHO-Klassifikation Patienten mit $\mathrm{PH}$ in 5 Gruppen eingeteilt: (1) Pulmonal-arterielle Hypertonie (PAH), (2) PH bei Erkrankungen des linken Herzens, (3) PH bei Lungenerkrankungen und/oder Hypoxie, (4) PH aufgrund chronischer Thromboembolien (CTEPH) und (5) PH mit unklaren multifaktoriellen Mechanismen [1]. Die WHO-Gruppe 1 (PAH), definiert als Erkrankung mit einem mittleren PAP $>20 \mathrm{mmHg}$, normalem Druck im linken Vorhof und einem pulmonalen Gefäßwiderstand $\geq 3$ Wood-Einheiten (WU) [1], wird, nach unterschiedlichen Grunderkrankungen bzw. pathophysiologischen Mechanismen, weiter in verschieden Untergruppen unterteilt. Eine hereditäre PAH (HPAH) liegt bei Patienten mit einer familiären Vorgeschichte oder nachgewiesener Keimbahnmutationen vor. Eine solche vererbte Suszeptibilität wird nicht nur durch Mutationen des Gens BMPR2 (bone morphogenetic protein receptor type II), sondern auch durch neu identifizierte Mutationen in neuartigen kausalen Genen vermittelt [3]. PAH kann auch durch bestimmte Wirkstoffe und Toxine induziert werden [4]. Durch andere Ursachen bedingte PAH sind beispielsweise die so genannte APAH (assoziierte PAH), PAH bedingt durch einen angeborenen Herzfehler (congenital heart disease, CHD), v.a. Ventrikelseptumdefekt, Vorhofseptumdefekt und durchgängigen Ductus arteriosus, PAH bedingt durch eine Lebererkrankung (portopulmonale Hypertension), PAH bedingt durch eine HIV-Infektion und PAH bedingt durch Schistosomiasis. PAH kann außerdem mit Autoimmunerkrankungen einhergehen, insbesondere mit systemischer Sklerose (SSc, Prävalenz 10\%-15\%), Mischkollagenosen (mixed connective tissue diseases, MCTD) und systemischem Lupus erythematodes (SLE). In der WHO-Gruppe 1 werden 2 weitere Krankheitsbilder der PAH abgegrenzt: die pulmonale venookklusive Erkrankung (pulmonary veno-occlusive disease, PVOD), die erblich (Mutation des Gens EIF2AK4 (eukaryotic translation initiation factor $2 \alpha$ kinase)), mit Autoimmunerkrankungen assoziiert oder idiopathisch sein kann, sowie die persistierende pulmonale Hypertonie des Neugeborenen (persistent pulmonary hypertension of the newborn, PPHN). PPHN kann idiopathisch sein oder durch mehrere verschiedene Lungenerkrankungen verursacht werden. Für die größte Gruppe der PAH-Erkrankungen, die idiopathische PAH (IPAH), wurden bislang keine Ursachen oder assoziierten Erkrankungen nachgewiesen. Patienten der WHOGruppe 4 (CTEPH) lassen sich weiter danach unterscheiden, ob sie operiert werden können (für eine pulmonale Endarteriektomie [PEA] oder eine pulmonale Ballonangioplastie (balloon pulmonary angioplasty, BPA) in Frage kommen) oder inoperabel sind.

Die aktuellen PAH-spezifischen Wirkstoffe zielen in erster Linie auf eine Dilatation des pulmonal-arteriellen Gefäßsystems ab [5]. Bei inoperablen Patienten mit CTEPH werden PAH-spezifische Wirkstoffe ebenfalls angewendet, um den erhöhten pulmonal vaskulären Druck einzustellen [2]. Die Prognose bleibt jedoch für Patienten mit PAH, selbst mit PAH-spezifischer Pharmakotherapie, weiterhin schlecht. Die durchschnittliche 5-Jahres-Überlebensrate beträgt für PAH 57\%-59\% [6,7] und für inoperable CTEPH 53\%-69\% [7, 8, 9]. Daher ist ein besserer Einblick in die Pathogenese von PAH und CTEPH dringend erforderlich, um neue Behandlungsstrategien entwickeln zu können.

Insbesondere für PAH zeigt ein wachsender Korpus an Evidenzdaten, dass Entzündungsprozesse möglicherweise eine Rolle bei der Pathobiologie der Erkrankung spielen [10]. Allerdings belegen aktuelle Studien, dass Entzündungszellen auch zum pathologischen Geschehen bei CTEPH beitragen könnten [11, 12]. Die vorliegende Übersichtsarbeit soll die wachsenden Erkenntnisse über Entzündungszellen im Rahmen der Pathogenese von PH sowie die Anwendung inflammatorischer Biomarker und immunmodulatorischer Therapien bei PAH und CTEPH zusammenfassen.

\section{Entzündung und Immunität bei PAH und CTEPH}

\section{Histopathologie bei PAH und CTEPH}

PAH und CTEPH sind gekennzeichnet durch ein vaskuläres Remodeling infolge erhöhter pulmonal-arterieller Drücke (Abb. 1). Merkmale des pulmonal-vaskulären Remodelings bei $\mathrm{PH}$ sind eine Verdickung der Intima und die Bildung obliterierender konzentrischer Läsionen in der Endothel- und/oder glatten Muskelzellschicht. In der Media, die hauptsächlich aus glatten Muskelzellen besteht, lässt sich ebenfalls eine Zunahme der Dicke beobachten. Interessanterweise korrelierte die kombinierte Dicke von Intima und Media signifikant mit dem PAP und dem pulmonal vaskulären Widerstand (PVR) [13]. Und schließlich waren eine erhöhte Dicke und ein Remodeling der Adventitia die auffälligsten Ergebnisse bei einer Reihe von 19 Autopsien der Lunge von IPAH-Patienten [14]. Diese erhöhte Dicke der Adventitia wurde jedoch in einer jüngsten Studie nicht bestätigt, was möglicherweise durch methodologische Unterschiede zwischen den beiden Studien erklärt werden kann [13].

Über die erhöhte Dicke von Intima, Media und Adventitia hinaus ist eine perivaskuläre Entzündung ein weiteres markantes Merkmal bei Patienten mit PAH. Ein Merkmal, das insbesondere bei Patienten mit IPAH zu beobachten ist, ist die Bildung plexiformer Läsionen. Diese werden üblicherweise als dynamische Geflechte 


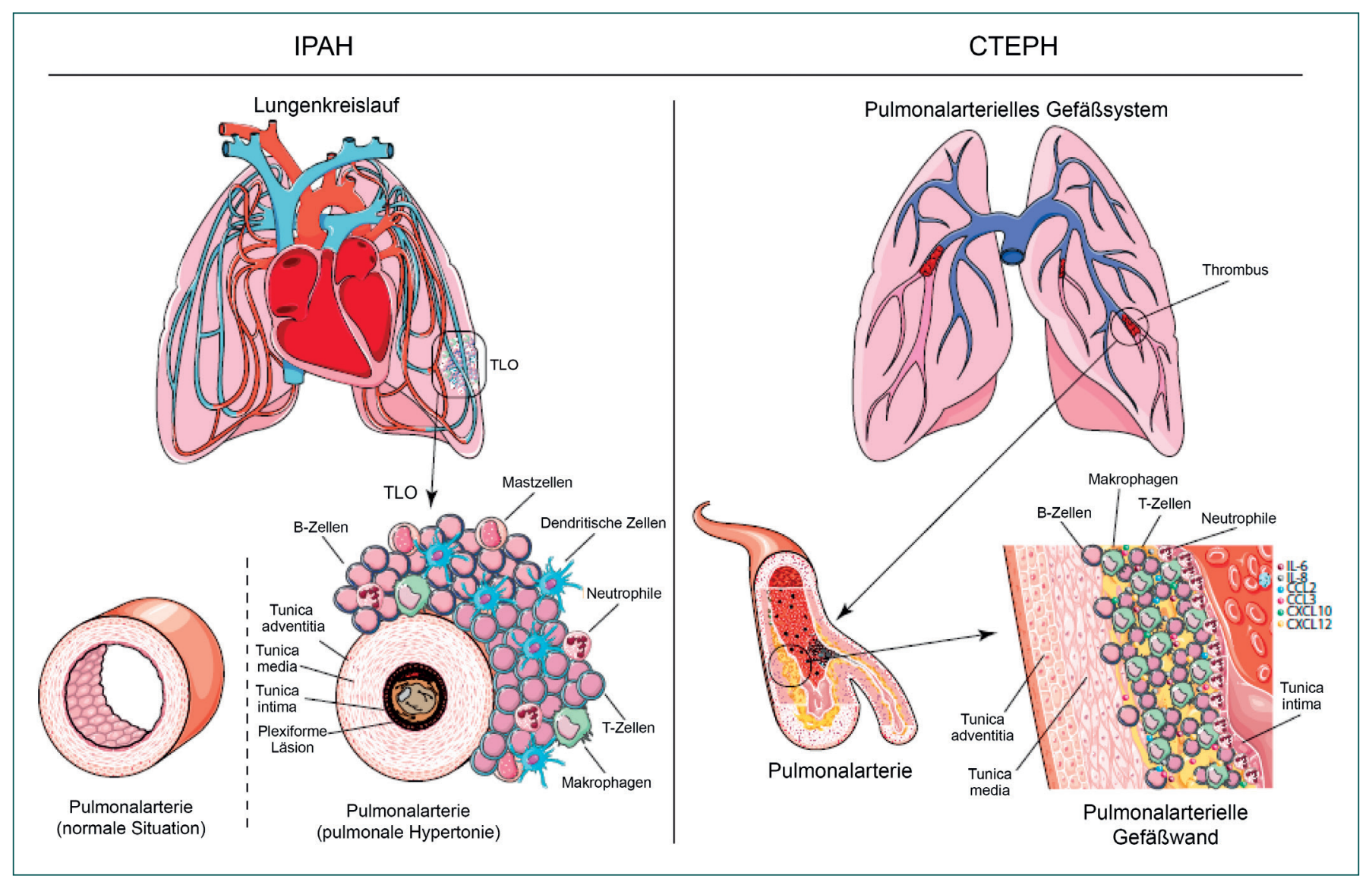

Abb. 1. Immunhistopathologie bei IPAH und CTEPH. Links: Schematische Übersicht über Zellen in tertiären lymphatischen Organen (TLO) bei Patienten mit idiopathischer pulmonal-arterieller Hypertonie (IPAH). Bei pulmonaler Hypertonie (PH) zeigt sich eine Hyperproliferation des Endothels in der Tunica intima mit Bildung geflechtartiger (plexiformer) Läsionen im Arterienlumen. Des Weiteren ist eine Hyperplasie der glatten Muskelzellen (smooth muscle cells, SMC) in der Tunica media der Pulmonalarterie erkennbar. Die Tunica adventitia ist umgeben von einer Kombination aus B-Zellen, T-Zellen, Mastzellen, dendritischen Zellen, Neutrophilen und Makrophagen. Rechts: Schematische Übersicht über vaskuläres Remodeling und Entzündung im thrombotischen Material von Patienten mit chronisch-thromboembolischer pulmonaler Hypertonie (CTEPH). Zwischen der (Neo-)Intima und der Tunica media ist ein Zustrom von Entzündungszellen wie B-Zellen, T-Zellen, Neutrophilen und Makrophagen erkennbar. Darüber hinaus liegen vermehrt proinflammatorische Mediatoren wie z.B. Interleukin (IL)-6, IL-8, die CC-Chemokin-Liganden 2 und 3 (CCL2 und CCL3) sowie die CXC-Chemokine 10 und 12 (CXCL10 und CXCL12) vor.

von Gefäßkanälen definiert und entstehen durch monoklonale Proliferation von Endothelzellen [15]. Histologische Untersuchungen bei Patienten mit CTEPH zeigen neo-intimale, thrombotische, rekanalisierte und atherosklerotische Läsionen im pulmonal-arteriellen Gefäßsystem. Diese chronisch-thrombotischenLäsionenbestehenausKollagen,Elastin,Entzündungszellen, rekanalisierten Gefäßen und kalzifiziertem Material [16]. Nach den aktuellen Konzepten handelt es sich bei CTEPH um eine duale Gefäßerkrankung mit Stenose, Netzen und Verschlüssen in den großen und mittelgroßen Pulmonalarterien (Pas) an den Stellen früherer Lungenemboli. Darüber hinaus zeigen Lungenbiopsien von Patienten mit CTEPH eine sekundäre Arteriopathie der kleinen Widerstandsgefäße [17].

Dysregulierte Immunantwort bei PAH und CTEPH

Es gibt zwei Formen von Immunreaktionen: die angeborene und die adaptive (erworbene) Immunität. Die angeborene Immunität ist unsere schnelle erste Verteidigungslinie gegen Mikroorganis- men und körperfremde Krankheitserreger, die sehr breit und unspezifisch ist. Die angeborene Immunantwort wird gewöhnlich von Makrophagen, Neutrophilen, natürlichen Killerzellen (NKZellen) und dendritischen Zellen (DC) induziert und durchgeführt. Parallel dazu wird die adaptive Immunreaktion initiiert, die eine sehr viel spezifischere Antwort durch T- und B-Lymphozyten ermöglicht. Neben ihrer angeborenen Funktion initiieren DCs adaptive Immunreaktionen, indem sie in lymphatische Organe einwandern, meist in die Lymphknoten, in denen die Lymphe aus dem Gewebe gefiltert wird. Hier präsentieren sie über MHC (major histocompatibility complex)-Moleküle Teile von Fremdpathogenen auf ihrer Zelloberfläche und induzieren so die klonale Expansion antigenspezifischer T-Zellen. T- und B-Gedächtniszellen können eine lebenslange Immunität vermitteln und ermöglichen dem Organismus, bei einem Zweitkontakt sehr viel rascher und effektiver auf einen Erreger zu reagieren. Die adaptive Immunantwort hängt von der Fähigkeit der T- und B-Zellen ab, zwischen körpereigenen und körperfremden Antigenen zu 
unterscheiden. Ist diese Fähigkeit gestört, kann es dazu kommen, dass das Immunsystem körpereigene Zellen angreift und so eine Autoimmunerkrankung auslöst. Elemente hiervon finden sich sowohl bei PAH als auch bei CTEPH. So ist eine IPAH etwa durch eine perivaskuläre Entzündung gekennzeichnet, bei der es zu einer pulmonal-lymphoiden Neogenese mit Bildung tertiärer lymphatischer Organe (TLO) kommt. Diese weisen spezifische Zonen von T- und B-Zellen auf, die vor Ort aktiviert werden und die Antikörperproduktion aufnehmen [18]. Neben den Lymphozyten können diese entzündlichen Läsionen auch dendritische Zellen, Makrophagen und Mastzellen enthalten [18, 19]. Darüber hinaus besteht eine positive Korrelation zwischen dem Grad der pulmonalen perivaskulären Entzündung und der Dicke von Intima/Media/Adventitia und dem mittleren pulmonal-arteriellen Druck (mean pulmonary arterial pressure, mPAP). Dies lässt darauf schließen, dass die Entzündung eine Rolle beim pulmonal vaskulären Remodeling und der Entwicklung von $\mathrm{PH}$ spielen könnte [20].

In thrombotischen und atherosklerotischen Läsionen von Patienten mit CTEPH wurden aktivierte B- und T-Lymphozyten, Makrophagen und Neutrophile nachgewiesen [21]. Im Rahmen der vorliegenden Studie ergaben topographische Analysen eine transmurale Verteilung von T-Zellen, während B-Zellen nur in geringer Zahl und meist tief im Inneren der Läsion nahe der inneren elastischen Lamina und nativen Media vorkamen. Auch wenn Entzündungszellen häufig in thrombotischem Material zu finden sind, ist deren Anreicherung möglicherweise ein Zeichen für eine Beteiligung am pathologischen Geschehen, d.h. der Nichtabheilung thrombotischer und atherosklerotischer Läsionen, bei Patienten mit CTEPH.

Im Folgenden diskutieren wir mehrere unterschiedliche Immunzellen des angeborenen bzw. adaptiven Immunsystems sowie ihre mögliche Bedeutung für die Pathogenese der PH.

\section{Angeborene Immunität}

\section{Makrophagen}

Makrophagen sind myeloische Leukozyten der ersten Verteidigungslinie, die sich in pulmonalen Läsionen von Patienten mit PH beobachten lassen $[22,23]$. Lungenmakrophagen werden klassischerweise in interstitielle und alveoläre Makrophagen unterteilt, wobei in aktuellen Studien mit Einzelzell-RNA-Sequenzierung und Zelllinien-Tracing eine Vielzahl von LungenmakrophagenSubtypen bei Mäusen identifiziert wurden [24, 25, 26]. In einem Hypoxie-induzierten $\mathrm{PH}-$ Mausmodell waren speziell die interstitiellen, nicht jedoch die alveolären, Makrophagen erhöht [27]. Über ihre Produktion von Zytokinen sind sie möglicherweise an der Entwicklung von PH beteiligt [28, 29, 30]. Des Weiteren entwickeln sich die bei PAH perivaskulär vorliegenden Lungenmakrophagen aus im Blut zirkulierenden peripheren Monozyten [31] Dies weist wahrscheinlich darauf hin, dass ihre Rekrutierung und Differenzierung auf ein Entzündungsgeschehen in der Lunge zurückzuführen ist. Chemokine, wie etwa CC-Chemokin-Ligand 2 (CCL2) und CX3C-Chemokin-Ligand 1 (CX3CL1), migrieren an die Stelle der Entzündung und differenzieren dort zu proinflammatorischen Makrophagen. Bei Patienten mit PH zeigten sich eine erhöhte Expression von Chemokinen, z.B. CCL1, CCL2, CCL5 und CX3CL1, in zirkulierenden Monozyten sowie erhöhte pulmonale Werte für CCl1, CCL2, CCL3, CLL3 und CX3CL1 [31]. Bei CX3CR1 (Rezeptor für CX3CL1)-defizienten Mäusen wurden nach Hypoxie-Exposition im Vergleich zu den Kontrollmäusen eine Verminderung sowohl der pulmonalen Entzündung als auch des vaskulären Remodelings beobachtet [31]. Interessanterweise fand man in pulmonalen vaskulären Endothelzellen von Patienten mit PAH eine übermäßige Expression von CCL2, der als Chemoattraktor für zirkulierende Entzündungszellen und als Wachstumsfaktor für pulmonal-arterielle glatte Muskelzellen (pulmonary arterial smooth muscle cells, PASMC) wirkt. Darüber hinaus zeigten PASMC und perivaskuläre Makrophagen von Patienten mit PAH im Vergleich zu Kontrollen erhöhte CCR2- und CCR5Werte $[32,33]$. Tatsächlich war die Expression von CCR2 und CCL5-CCR5 sowohl bei Makrophagen als auch bei PASMC erforderlich, um die Proliferation von PASMC zu initiieren und zu verstärken [33]. Bei der Differenzierung zirkulierender Monozyten zu interstitiellen Makrophagen bei Hypoxie-induzierter PH im Mausmodell exprimieren diese Thrombospondin-1 (TSP-1). Dies führt über die Aktivierung des transformierenden Wachstumsfaktors beta (TGF- $\beta$ ) zu einer Rho-Kinase-vermittelten Vasokonstriktion [34] und somit zu einer Verstärkung der PH-Pathologie. All diese Studien weisen auf eine möglicherweise maßgebliche Rolle der chemokinvermittelten Rekrutierung von Makrophagen in der frühen Pathogenese von PH hin.

Es wurde gezeigt, dass die Makrophagenrekrutierung und -aktivierung außerdem eine wichtige Rolle bei der Pathogenese von Hypoxie-induzierter PH im Mausmodell und in Sugen-/athymischen Rattenmodellen spielt $[35,36,37]$. In einer früheren Studie wird berichtet, dass bei Patienten mit PH Hypoxie-induzierbarer Faktor-1 $\alpha$ (HIF-1 $\alpha$ ) durch Lungenmakrophagen exprimiert wird, insbesondere in plexiformen Läsionen [38]. Bei Mäusen ohne HIF-1a wurden eine signifikante Verringerung des rechtsventrikulären (RV) systolischen Drucks (RVSP) und RV-Hypertrophie zusammen mit einer geringeren Infiltration von Makrophagen in die Lunge und rechte Herzkammer beobachtet, was auf eine direkte Wirkung lokal vorhandener Makrophagen bei der Induzierung von $\mathrm{PH}$ schließen lässt [39]. In der Lunge von Patienten mit IPAH fand sich eine höhere Zahl von Makrophagen und Monozyten als bei gesunden Kontrollen [40]. Ein weiterer Ph-induzierender Faktor, das Resistin-ähnliche Molekül- $\alpha$ (resistin-like molecule- $\alpha$, RELM $\alpha$ ) war in einem Hypoxie-induzierten Mausmodell für PH erhöht. Außerdem wurde festgestellt, dass sein humanes Homolog, Resistin, bei Patienten mit IPAH in Makrophagen-ähnlichen Entzündungszellen hochreguliert war. Bei diesen Patienten förderten Resistin-stimulierte Makrophagen die apoptoseresistente Proliferation von PASMC [41].

Makrophagen spielen möglicherweise auch eine Rolle bei anderen Formen von $\mathrm{PH}$, da proinflammatorische Makrophagen in erhöhter Zahl im Material von CTEPH-Patienten nach pulmonaler Endarteriektomie (PEA) gefunden wurden [21]. Darüber hinaus 
wurde im Serum von acht CTEPH-Patienten eine erhöhte Expression von MIP1a (macrophage inflammatory protein-1a, synonym: CCL3) nachgewiesen. Diese führt zur Synthese proinflammatorischer Zytokine, zu vaskulärem Remodeling und zur Rekrutierung von Makrophagen [42]. Vor kurzem gaben Florentin et al. und Pullamsetti et al. einen umfassenden Überblick über die zunehmende Anzahl von Belegen für die Rolle von Makrophagen bei PH [43, 44]. Zusammengefasst spielen Makrophagen aufgrund der Produktion proinflammatorischer Zytokine sowie der Initiierung und Proliferation von PASMC und Hypoxiefaktoren eine Schlüsselrolle bei der Pathogenese von $\mathrm{PH}$.

\section{Neutrophile}

Neutrophile zählen zu den früh reagierenden Komponenten des Immunsystems und werden durch Chemokine, die von gewebsständigen Immunzellen (z.B. Makrophagen) produziert werden, bei einer akuten Entzündung an den Ort des Geschehens rekrutiert. Neutrophile sind bekannte Phagozyten und können Mikroorganismen oder Partikel verdauen. Neben ihrer phagozytierenden Funktion sind Neutrophile in der Lage, antimikrobielle Inhaltsstoffe zu degranulieren und freizusetzen. Bei Patienten mit PAH korreliert ein erhöhtes Verhältnis von Neutrophilen zu Lymphozyten in Proben peripheren Blutes positiv mit der funktionellen NYHA-Klassifikation, und ein negatives Verhältnis ist sogar ein Prädiktor für ereignisfreies Überleben $[45,46]$. Bei Patienten mit CTEPH könnte das Neutrophilen-Lymphozyten-Verhältnis ein Prädiktor für die postoperative Sterblichkeit sein und als nicht invasives Messinstrument für eine Stratifizierung des Operationsrisiko herangezogen werden [47]. Aktuell ist unklar, ob und wie Neutrophile zur Progredienz der PAH beitragen. In murinen Modellen reicherten sich Neutrophile bei Ratten mit hypoxischer $\mathrm{PH}$ und mit Monocrotalin (MCT)-induzierter PH in der Lunge am Ort der Entzündung/Schädigung an [36, 48]. Aktuelle Evidenzdaten lassen darauf schließen, dass die Produktion von Myeloperoxidase (MPO), einem Katalysator für die Bildung reaktiver Sauerstoffspezies (ROS), durch Neutrophile eine Krankheitsprogression verursachen kann [49]. Die MPO-Plasmaspiegel waren bei Patienten mit PAH im Vergleich zu gesunden Kontrollen erhöht. Des Weiteren zeigten Hypoxie-exponierte Mpo-/Mäuse einen niedrigeren Anstieg des rechtsventrikulären Drucks als Wildtyp-Mäuse [49], was aufgrund der Produktion von MPO und einer ungünstigen Funktion der pulmonalen Gefäße auf eine pathogene Bedeutung der Neutrophilen bei $\mathrm{PH}$ hindeutet.

\section{Mastzellen}

Mastzellen (mast cells, MC) sind langlebige gewebsständige Immunzellen, die bekanntermaßen aufgrund der Freisetzung von Histamin und der Produktion proinflammatorischer Zytokine eine wichtige Rolle für das Immunsystem spielen. Darüber hinaus sind Mastzellen auch für ihre Rolle im Rahmen der Angiogenese durch die Produktion von Endothelwachstumsfaktor (vascular endothelial growth factor, VEGF) und Proteinasen, u.a. Chymase und Tryptase, bekannt [50, 51, 52]. Eine zunehmende Zahl von Belegen legt nahe, dass Mastzellen eine Rolle im Rahmen der Pa- thophysiologie der PH spielen. Mastzellen kommen bei Patienten mit IPAH in entzündlichen Läsionen vor [19], sogar in frühen perivaskulären Zellläsionen in der Lunge dieser Patienten [53]. In den letzten Jahren wurde die Rolle von Mastzellen im Rahmen von Pilotversuchen (proof-of-principle) gezeigt. Bei Ws/Ws-Mäusen, die aufgrund einer Mutation des Mastzellen-Wachstumsfaktor-Rezeptors c-kit keine Mastzellen besitzen [54], waren die Merkmale einer experimentellen PH, z.B. RVSP, PVR, RV Hypertrophie und vaskuläres Remodeling, nach PulmonalarterienBanding oder Behandlung mit MCT weitgehend abgeschwächt [55]. Durch eine Inhibition der Degranulation von Mastzellen mittels Ketotifen konnte außerdem die Entwicklung von $\mathrm{PH}$ in verschiedenen experimentellen $\mathrm{PH}$-Rattenmodellen reduziert werden $[55,56,57,58]$. In einer kleinen klinischen Studie wurden 9 Patienten mit PAH mit Cromolyn und Fexofenadin, die beide die Mastzellaktivität hemmen, behandelt. Bei diesen Patienten wurden eine Verringerung der VEGF-Spiegel und der zirkulierenden proangiogenen myeloiden Zellen sowie ein Anstieg des ausgeatmeten Stickoxids (bei PAH in der Regel niedrig) beobachtet, was darauf schließen lässt, dass die Behandlung eine supprimierende Wirkung auf Mastzellen haben könnte [59]. Aus mechanistischer Sicht geht man davon aus, dass Mastzellenproteasen eine wichtige Rolle im Hinblick auf die Entwicklung von PH und ihren Schweregrad spielen. In Lungengewebe quantitativ bestimmte Mastzellenproteasen wie Chymase und Tryptase korrelieren mit dem Schweregrad der PH und des pulmonal-vaskulären Remodelings [52, 60, 61, 62, 63]. Darüber hinaus wurde im Lungengewebe von MCT-Ratten, nicht aber in der rechten Herzkammer, eine unverhältnismäßig starke Infiltration und Degranulation von Mastzellen nachgewiesen. Dies weist auf eine Freisetzung von Proteasen, z.B. Tryptase, hin, die zum pulmonal-vaskulären Remodeling beiträgt [64]. Und schließlich lassen sich bei MCT-getesteten Ratten Mastzellen um die distalen Pulmonalarterien herum sowie eine Anreicherung von Makrophagen beobachten, was darauf hindeutet, dass Mastzellen am vaskulären Remodeling in der Lunge beteiligt sind [64]. Zusammengefasst spielen Mastzellen eine pathobiologische Rolle bei $\mathrm{PH}$, höchstwahrscheinlich über die Freisetzung von proangiogenen Faktoren und Mastzellenproteasen.

\section{Natürliche Killerzellen}

Natürliche Killer (NK)-Zellen sind wichtige Komponenten des angeborenen Immunsystems, da sie schnell auf virusinfizierte Zellen reagieren. Außerdem weiß man, dass diese Zellen mit zytotoxischer Aktivität die Angiogenese und das vaskuläre Remodeling regulieren. Die mögliche Rolle von NK-Zellen im Rahmen der Pathogenese von PAH und CTEPH wurde in wenigen Studien beurteilt. Allerdings wurden sowohl bei Patienten mit PAH als auch in experimentellen PH-Modellen mit Nagern eine verminderte Zahl von NK-Zellen und verminderte Zytotoxizität festgestellt [65]. Des Weiteren wurden in 2 unabhängigen genetischen Mausmodellen für eine Dysfunktion von NK-Zellen, bei denen den Tieren der Transkriptionsfaktor NFIL3 bzw. NK-aktivierender Rezeptor Nkp46 fehlte, erhöhte RV systolische Drücke und
130

Kompass Pneumol 2020;8:126-138 DOI: $10.1159 / 000507909$ 

RV Hypertrophie beobachtet. In beiden Modellen war diese Entwicklung der experimentellen $\mathrm{PH}$ an eine erhöhte Produktion von Interleukin-23 (IL-23) gekoppelt. Möglicherweise lag eine verminderte Zahl von NK-Zellen vor, die zu der erhöhten Produktion von IL-23 durch Lungenmakrophagen und andere myeloide Zelltypen führte [66]. Interessanterweise ist IL-23 für die Produktion proinflammatorischer Zytokine wie IL-17A/F, IL-21 und IL-22 sowie die Steuerung der Entwicklung naiver T-Zellen zu T-Helferzellen des Phänotyps TH17 bekannt [67]. Zusammengefasst tragen NK-Zell-Defekte möglicherweise über die falsche Regulierung des pulmonal-vaskulären Remodelings zur Pathogenese von PAH bei. Allerdings sind weitere Untersuchungen erforderlich, um diese Ergebnisse zu evaluieren.

\section{Verknüpfung von angeborener und adaptiver Immunität}

\section{Dendritische Zellen}

Dendritische Zellen sind zentrale Modulatoren zwischen Toleranz und Immunität. Man weiß, dass sie als Bindeglied zwischen angeborener und adaptiver Immunität fungieren. Die Hauptfunktion dendritischer Zellen besteht darin, Antigene aufzunehmen, zu prozessieren und gegenüber T-Zellen zu präsentieren. Dendritische Zellen können entweder durch mikrobielle Stimuli über sogenannte Pattern-Recognition-Rezeptoren, wie z.B. Tolllike-Rezeptoren (TLR), oder durch proinflammatorische Zytokine aktiviert werden. Dies führt zur Aktivierung des Nf- $\kappa \beta$ (nuclear factor kappa-light-chain-enhancer of activated B cells)-Signalwegs. Ferner regulieren dendritische Zellen kostimulatorische Moleküle hoch, produzieren verschiedene proinflammatorische Zytokine wie Interleukin (IL)- 6 und IL-12 und fördern - neben der Antigenpräsentation - die Aktivierung, Expansion und Differenzierung von T-Zellen $[68,69,70]$. Dieser Vorgang muss engmaschig kontrolliert werden, da eine fortgesetzte Aktivierung dendritischer Zellen zu schweren Nebenwirkungen, etwa zur Präsentation von Autoantigenen gegenüber T-Zellen, und damit zur Entwicklung von Autoimmunerkrankungen führen könnte [69, 71]. Theoretisch könnten dendritische Zellen auch bei der Pathophysiologie der PAH eine Rolle spielen [72].

Bei Patienten mit IPAH kommen dendritische Zellen vermehrt in der Lunge vor und lagern sich dort insbesondere um Pulmonalarterien an, bei denen es zu einem Remodeling gekommen ist [73]. Im Lungenparenchym werden hingegen meist unreife DCs beobachtet, was sich in einer erhöhten Zahl von DC-SIGN (dendritic cell-specific intercellular adhesion molecule 3-grabbing nonintegrin)-Rezeptoren und dendritischen Zellen zeigt [73]. Dendritische Zellen (DCs) lassen sich im Wesentlichen in 4 Subtypen unterteilen: konventionelle DCs vom Typ I (cDC1), die eine effiziente Kreuzpräsentation (d.h. die Präsentation exogener Antigene im Kontext von MHC-Klasse I und Induzierung der Antwort von CD8+-T-Zellen) bewirken, cDCs vom Typ II (cDC2), die in der Lage sind, die Antwort von CD4+-T-Zellen zu induzieren, plasmazytoide DCs (pDCs), die große Mengen an Typ-I-Interferon zur Bekämpfung von Virusinfektionen produzieren können sowie schließlich aus Monozyten hervorgegangene DCs (mono- cyte-derived DCs, mo-DCs oder myeloide dendritische Zellen, myeloid dendritic cells (mDCs)), die bei einer Entzündung aktiviert werden und große Mengen Chemokine produzieren, durch die T-Zellen zum Ort des Entzündungsgeschehens rekrutiert werden [74].

Die Anzahl der cDCs und pDCs war sowohl in Lungenzellsuspensionen insgesamt (periphere oder perihiläre Proben) als auch in den größeren Pulmonalarterien von Patienten mit IPAH im Vergleich zu denjenigen von Kontrollen erhöht [40]. Konfokalmikroskopische Analysen haben ergeben, dass sich pDCs hauptsächlich im Alveolarbereich in der Umgebung von Blutgefäßen finden. Im Gegensatz dazu war die Zahl der cDCs im peripheren Blut von Patienten mit IPAH reduziert [75]. Zusammen mit der erhöhten Zahl pulmonaler cDCs lässt dies auf eine Migration in die Lungen schließen. Derzeit liegen keine Daten für unterschiedliche cDCSubpopulationen bei der Pathogenese von PAH vor. Zusammengefasst spielen DCs eine entscheidende Rolle für das Auslösen adaptiver Immunantworten und könnten über die Antigenpräsentation und Produktion proinflammatorischer Zytokine an der Pathogenese von PAH beteiligt sein.

\section{Adaptive Immunität}

\section{T-Zellen}

T-Zellen sind ein sehr wichtiger Teil des adaptiven Immunsystems. CD4+-T-Zellen, auch als T-Helfer (Th)-Zellen bezeichnet, helfen dem Organismus durch das indirekte Zerstören von Erregern, indem sie die Aktivierung anderer Zellen des Immunsystems, z.B. B-Zellen, unterstützen. CD8+-T-Zellen, auch zytotoxische T-Zellen genannt, sind in der Lage Pathogene direkt abzutöten. Hierzu setzen sie Granzyme, die eine Apoptose induzieren, sowie das Protein Perforin frei, das in der Membran der Zielzelle Poren bildet. Gamma-delta $(\gamma \delta)$-T-Zellen stellen eine kleine Subpopulation von T-Zellen dar, die sich durch die Expression heterodimerer T-Zell-Rezeptoren (T-cell receptors, TCRs) bestehend aus $\gamma$ - und $\delta$-Ketten auszeichnen. Bei Lungenbiopsien von IPAHPatienten wurde mittels Flusszytometrie eine erhöhte Anzahl von CD4+-, CD8+-T-Zellen und $\gamma \delta$-T-Zellen in der Umgebung der Pulmonalarterien nachgewiesen [40]. Diese CD4+- und CD8+-TZellen liegen bei Patienten mit IPAH im Adventitiaraum um die Pulmonalgefäße herum vor [19]. Bei Patienten mit Schistosomiasis-assoziierter PAH und IPAH wurde ebenfalls eine erhöhte Anzahl periarterieller CD4+-T-Zellen nachgewiesen [76].

Man weiß, dass Th-Zellen eine tragende Rolle bei vielen Entzündungs- und Autoimmunerkrankungen spielen [77]. Th-Zellen lassen sich grob in Th1-, Th2- und Th17-Zellen unterteilen. In den pulmonalen TLOs von Patienten mit IPAH finden sich insbesondere Th17-Zellen [18]. Th17-Zellen sind die Hauptproduzenten von IL-17, IL-21 und IL-22. In durch Remodeling umgebauten Pulmonalarterien von IPAH-Patienten finden sich IL-21+-Zellen [78]. Naive Th-Zellen differenzieren in Anwesenheit von IL-1 $\beta$, IL- 6 und TGF- $\beta$ zu Th17-Zellen [79]. Bei Patienten mit IPAH sind die Serumspiegel von IL-1 $\beta$ und IL- 6 im Vergleich zu Kontrollen erhöht [80]. Bei Patienten, die an Bindegewebskrankheit-assozi-
132

Kompass Pneumol 2020;8:126-138 DOI: $10.1159 / 000507909$ 
ierter PAH (CTD-PAH) litten, war die Anzahl von Th17-Zellen und Th17-bezogenen Zytokinen im Vergleich zu gesunden Kontrollen erhöht [81]. Arbeiten unserer Gruppe zeigen, dass die TZell-Differenzierung durch den Grad der Expression von TNFAIP3 (tumor necrosis factor alpha (TNF- $\alpha$ )-induced protein 3 ) in DCs gesteuert wird, da TNFAIP3-defiziente DCs die Differenzierung von Th17-Zellen durch eine erhöhte Expression von IL$1 \beta$, IL- 6 und IL-23 stimulieren $[71,82]$. Eine DC-spezifische Deletion des TNFAIP3-Gens führt außerdem zu erhöhten NF-kBWerten, wodurch ein proinflammatorisches Milieu entsteht.

Follikuläre T-Helfer (Tfh)-Zellen exprimieren den Chemokinrezeptor CXCR5, was zu ihrer Lokalisierung in B-Zell-Follikeln beiträgt. Unter Einwirkung von IL-21, IL-6, IL-12 und IL-27 können sie aktivierte B-Zellen unterstützen, wodurch eine humorale Immunantwort induziert wird. In den TLOs von Patienten mit IPAH wurde eine Zunahme von IL-21, PD-1 und Tfh-Zellen festgestellt [18]. Bei Patienten mit CTEPH ergab die histologische Auswertung von PEA-Material eine Anreicherung von CD3+-Zellen in atherosklerotischen und thrombotischen Läsionen [21]. Man weiß wenig über die Unterschiede der T-Zell-Funktion bei CTEPH. Weitere Forschungsanstrengungen sind erforderlich, um Evidenzdaten bezüglich einer möglichen Rolle von T-Zellen im Rahmen der Pathogenese von CTEPH zu generieren. Zusammengefasst findet sich eine erhöhte Zahl von T-Zellen in der Lunge von Patienten mit IPAH sowie im PEA-Material von Patienten mit CTEPH. Scheinbar liegt bei PAH eine dysregulierte Th17Immunantwort vor; allerdings sind weitere Studien vonnöten, um diese Frage eingehender zu untersuchen.

\section{B-Zellen und humorale Immunantwort}

B-Zellen sind die Effektoren der humoralen Immunantwort. Nach der Antigenerkennung durch den B-Zell-Rezeptor können sie Antigene präsentieren, Zytokine sezernieren und zu Gedächtnis- oder Plasmazellen differenzieren, die große Mengen von Antikörpern produzieren. Nach ihrer Aktivierung sind B- und TZellen an einer Keimzentrumreaktion beteiligt, bei der Tfh-Zellen ihr Standardzytokin IL-21 produzieren. Hierdurch werden das Überleben, die Proliferation und Differenzierung von B-Zellen unterstützt. Darüber hinaus exprimieren aktivierte T-Zellen CD40L, das mit seinem Rezeptor CD40 in B-Zellen interagiert und so ein kostimulatorisches Signal gibt, das für die Aktivierung von B-Zellen und die Keimzentrumbildung von entscheidender Bedeutung ist. Wie oben erwähnt finden sich in der Lunge von PAH-Patienten TLOs, die B-Zellen, T-Zellen und dendritische Zellen enthalten. Diese hoch organisierten Strukturen weisen Hochendothelvenolen auf, die zirkulierenden Lymphozyten und Stromazellen, u.a. follikulären dendritischen Zellen, die mittels Fc-Rezeptoren Antigene gegenüber B-Zellen präsentieren, ein direktes Eindringen ermöglichen [83]. Zudem liefert das Vorliegen von IL-21 und Tfh-Zellen, B-Zellen, die aktivierungsinduzierte Cytidindesaminase exprimieren, welche für Klassenwechsel der schweren Kette von Immunglobulinen (immunglobulin heavy chain class switch) und die Affinitätsreifung von Antikörpern unverzichtbar ist, sowie Plasmazellen Belege für eine lokale, kon- tinuierliche Antikörperproduktion [18]. Über das Vorhandensein von TLOs in der Lunge hinaus gibt es zusätzliche Belege, welche die These einer dysregulierten B-Zell-Aktivierung bei IPAH und bei Bindegewebskrankheit-assoziierter PAH (CTD-PAH) stützen $[84,85]$. Zum ersten finden sich bei Patienten mit IPAH vermehrt zirkulierende Plasmablasten [86]. Zweitens liegen bei rund 40\% der Patienten mit IPAH Autoantikörper vor [87]. Diese Autoantikörper werden möglicherweise von den Plasmazellen im Inneren von TLOs in der Lunge von IPAH-Patienten produziert $[18,86]$ und erkennen Oberflächenantigene auf Endothelzellen [88]. Gegen Endothelzellen gerichtete Autoantikörper fördern die Apoptose von Endothelzellen und tragen so zum vaskulären Remodeling bei [86]. Darüber hinaus kann endothelspezifisches IgA die Zytokinproduktion und die Hochregulierung von Adhäsionsmolekülen durch Endothelzellen unterstützen [86, 88, 89, 90]. Gegen Endothelzellen gerichtete IgG-Antikörper versetzen Endothelzellen in einen proadhäsiven und proinflammatorischen Zustand [91]. In tierexperimentellen Studien führt die Injektion von Autoantikörpern von Patienten mit CTD-PAH bei gesunden Mäusen zu einer höheren Zahl glatter Muskelzellen in den Gefäßen und Atemwegen und zu einer entzündlichen Erkrankung der Lungengefäße [92]. Bei MCT-Ratten fand man hohe Plasmaspiegel von IgG, das Lungengefäßproteine markierte. Außerdem führte die Übertragung von Autoantikörpern an Ratten zu pulmonal-vaskulärem Remodeling und pulmonaler Hypertonie [93].

Bei CTEPH ist nur wenig über zirkulierende und thrombusständige B-Zellen bekannt. Eine Splenektomie ist ein allgemein bekannter Risikofaktor für CTEPH. In Anbetracht der Tatsache, dass die Milz für die Reifung von B-Zellen von Bedeutung ist, könnten pathogene B-Zellen eine Rolle bei der Pathogenese von CTEPH spielen. Derzeit laufen Studien, in denen periphere mononukleäre Blutzellen (peripheral blood mononuclear cells, PBMCs) von Patienten mit CTEPH massenzytometrisch untersucht werden. Die Ergebnisse dieser Studien stehen noch aus.

Zusammengefasst finden sich bei Patienten mit IPAH Autoantikörper, die spezifisch gegen Oberflächenantigene auf Endothelzellen gerichtet sind. B-Zellen sowie eine im Vorfeld stattfindende Bildung von Plasmazellen könnten eine wichtige Rolle bei der Pathogenese von PAH spielen.

\section{Diagnostische und prognostische inflammatorische Bio- marker bei PAH und CTEPH}

Inflammatorische Biomarker können möglicherweise bei PAH und CTEPH als diagnostische und prognostische Werkzeuge von Nutzen sein [21, 32, 42, 80, 94, 95, 96, 97, 98, 99, 100, 101, 102]; diese sind in Tabelle 1 dargestellt. Proinflammatorische Zytokine und Chemokine können unmittelbar zur Rekrutierung von Immunzellen, zur Aktivierung und Proliferation von PASMCs sowie zu endothelialer Dysfunktion beitragen. Das bislang bedeutendste Zytokin scheint IL-6 zu sein, das in vielfältigem Zusammenhang mit der Pathogenese von PAH steht. In tierexperimentellen Modellen wurde im Anschluss an die Verabreichung von rekombinantem IL-6 sowie in IL-6-transgenen Mäusen die Entwick- 


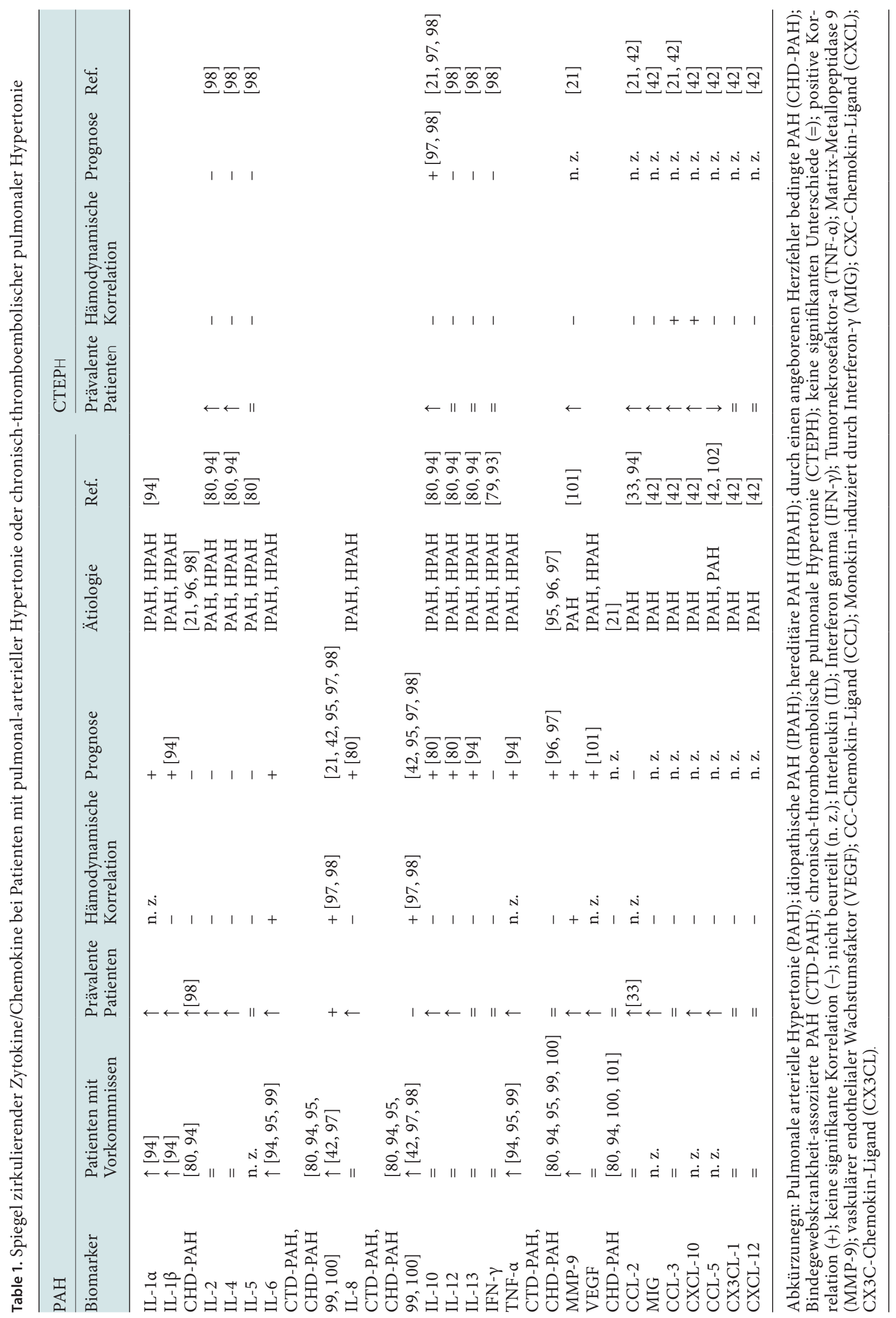


lung von PH beobachtet [103, 104, 105], während sich IL-6-Knockout-Mäuse resistent gegen Hypoxie-induzierte PH zeigten [106]. In klinischen Studien korrelierte IL-6 nachweislich mit dem Überleben und der Lebensqualität von Patienten mit IPAH [80, 107] und war prädiktiv für das Langzeitansprechen von CTEPHPatienten auf eine PEA [98]. In einer aktuellen Studie wurden bei Patienten mit PAH mit Hilfe von maschinellem Lernen ZytokinCluster generiert. Die Analysen zeigten, dass die immunen Phänotypen sich nicht nach den PAH-Subtypen der Gruppe 1 gemäß WHO-Klassifikation [108] richteten. Dies weist darauf hin, dass immune Phänotypen innerhalb der Subtypen der WHO-Gruppe 1 variieren könnten. Ferner bieten diese Ergebnisse möglicherweise einen Rahmen, um das Ansprechen von Patienten auf neue Therapien, die auf eine Immunität abzielen, zukünftig zu untersuchen.

Bei Mäusen mit einer Überexpression von Tumornekrosefaktor alpha (TNF- $\alpha$ ) wurde die Spontanentwicklung von PH beobachtet [109]. In einer klinischen Studie mit CTEPH-Patienten, die sich einer PEA unterzogen, wurden vor dem Eingriff erhöhte Spiegel von TNF- $\alpha$, IL-6 und IL-10 festgestellt [97]. Für IL-6 und IL-10 wurden unmittelbar nach dem Eingriff Spitzenspiegel nachgewiesen, während der TNF- $\alpha$-Spiegel innerhalb der ersten 24 Stunden nach der PEA signifikant sank [97].

Bei nicht vorbehandelten Patienten mit CTD-PAH waren die Ausgangswerte des Plazenta-Wachstumsfaktors (placental growth factor, PlGF), von sVEGFR-1, TNF- $\alpha$ und VEGF-D erhöht und stellten ein Differenzierungsmerkmal zwischen gesunden Kontrollen/Patienten mit IPAH und Patienten mit CTD-PAH dar. Nach viermonatiger gezielter Behandlung gegen $\mathrm{PAH}$ waren darüber hinaus die sVEGFR-1-Spiegel gesunken, was darauf hinweist, dass es sich bei diesem Wachstumsfaktor um einen Parameter handelt, dessen Bestimmung unter Therapien lohnenswert ist [99]. In einer Studie mit 206 PAH-Patienten waren die Spiegel von Angiopoietin 1 (Ang-1), VEGF und Matrix-Metallopeptidase 9 (MMP-9) zum Follow-up-Zeitpunkt 16 Wochen nach Baseline mit einem erhöhten Risiko für Tod und Hospitalisierung assoziiert [101]. Eine Vielzahl der inflammatorischen Biomarker wird derzeit noch erforscht, da weitere präklinische, translationale und klinische Studien erforderlich sind, um den klinischen und prognostischen Wert dieser Marker zu bestimmen.

\section{Immunmodulatorische Therapie bei PAH und CTEPH}

Viel versprechende neuartige inflammatorische Zielmoleküle für Therapien und laufende klinische Studien zur Beurteilung möglicher therapeutischer Wirkstoffe sind in Abbildung 2 dargestellt. Daneben gibt die ergänzende Tabelle S1 (Original online verfügbar unter https://www.mdpi.com/2077-0383/9/2/561/s1) einen Überblick über die Abschwächung einer experimentellen $\mathrm{PH}$ durch gezielte Beeinflussung immunmodulatorischer Signalwege.

Gestützt auf die oben dargestellten Evidenzdaten ist das Targeting immunologischer und inflammatorischer Signalwege unter Umständen ausreichend, um die Erkrankung zu behandeln und ein Fortschreiten zu verhindern. Frühere Studien haben ergeben, dass im inflammatorischen MCT-Rattenmodell für PH der PHPhänotyp durch entzündungshemmende Wirkstoffe, z.B. Dexamethason und Mycophenolatmofetil, sowie eine Inhibition des NFAT (nuclear factor of activated T cell) mittels Cyclosporin verhütet und revertiert werden kann [110, 111, 112]. Bei Patienten mit SLE und MCTD-PAH senkte eine Kombinationsbehandlung mit Cyclophosphamid und Glukokortikoiden möglicherweise wirksam den PVR bei Patienten mit weniger schwerer PH bei Baseline [113].

Ein gezielterer Therapieansatz, wie etwa die Behandlung mit antiIL-1, verhinderte nachweislich den PH-Phänotyp bei Ratten mit MCT-PH [114]. Eine aktuelle Studie der Virginia Commonwealth University zur Beurteilung der Wirksamkeit einer Behandlung von PAH-Patienten mit Anakinra, einem IL-1-Rezeptor-Antagonisten, steht kurz vor dem Abschluss.

In neueren Studien führte die Behandlung mit einem IL-6-spezifischen Antagonisten zur Reversion einer experimentellen $\mathrm{PH}$ in Rattenmodellen mit MCT-PH und Sugen/Hypoxie (SU/Hx)-induzierter PH [115]. Bei Mäusen mit Hypoxie-induzierter PH wurde auch die abschwächende Wirkung einer IL-6-Blockade festgestellt [78]. Patienten mit PAH werden im Rahmen der aktuell laufenden Studie TRANSFORM-UK mit anti-IL-6 behandelt werden. Die Ergebnisse der Studie werden in Kürze erwartet [116].

Bei Mäusen mit Hypoxie-induzierter PH wurde eine erhöhte Expression von IL-17 und IL-21, Signaturgene für Th17- bzw. TfhZellen, festgestellt [78]. Während die Blockade von IL-17 keinerlei Wirkungen auf den RVSP und die RV Hypertrophie zeigte, waren IL-21-Rezeptor-Knockout-Mäuse gegen Hypoxie-induzierte PH resistent [78]. Eine verstärkte Expression von M2-MakrophagenMarkern und IL-21, das Makrophagen in Richtung eines M2Phänotyps polarisieren kann, wurde in der Lunge von Patienten mit IPAH gefunden, bei denen eine Lungentransplantation durchgeführt wurde. Zusammen mit der bekannten herausragenden Rolle, die IL-21 für die Interaktion zwischen B- und T-Zellen spielt, weisen diese Ergebnisse auf IL-21 als potenziellen Angriffspunkt für eine Behandlung von PAH hin [78]. Bei experimenteller Autoimmunarthritis zeigte eine kombinierte IL-6/IL-21-Blockade günstige synergistische Wirkungen im Zusammenhang mit einer stark verminderten Th17-Differenzierung [117].

Die Behandlung mit einem TNF- $\alpha$-Hemmer (Etanercept) führte sowohl bei Ratten mit MCT-PH [118, 119] als auch bei Ratten mit SU/Hx-induzierter PH nachweislich zu einer Abschwächung des PH-Phänotyps [120]. Bei endotoxämischen Schweinen führte eine Anti-TNF- $\alpha$-Therapie zur Reversion des PAH-Phänotyps [121]. Derzeit liegen keine (prä-)klinischen Studien zur Bestimmung der möglichen klinischen Wirkungen von Etanercept bei Patienten vor.

Vermehrte Kenntnisse über Funktionsverlustmutationen (Lossof-Function-Mutationen) im BMPR2-Signalweg haben zur Aufnahme von Studien zur Beurteilung möglicher neuartiger therapeutischer Zielmoleküle in dieser Kaskade geführt. BMPR2, das hauptsächlich in vaskulären Endothelzellen exprimiert wird [122], gehört zur Familie der TGF- $\beta$-Rezeptoren. Zahlreiche Stu- 


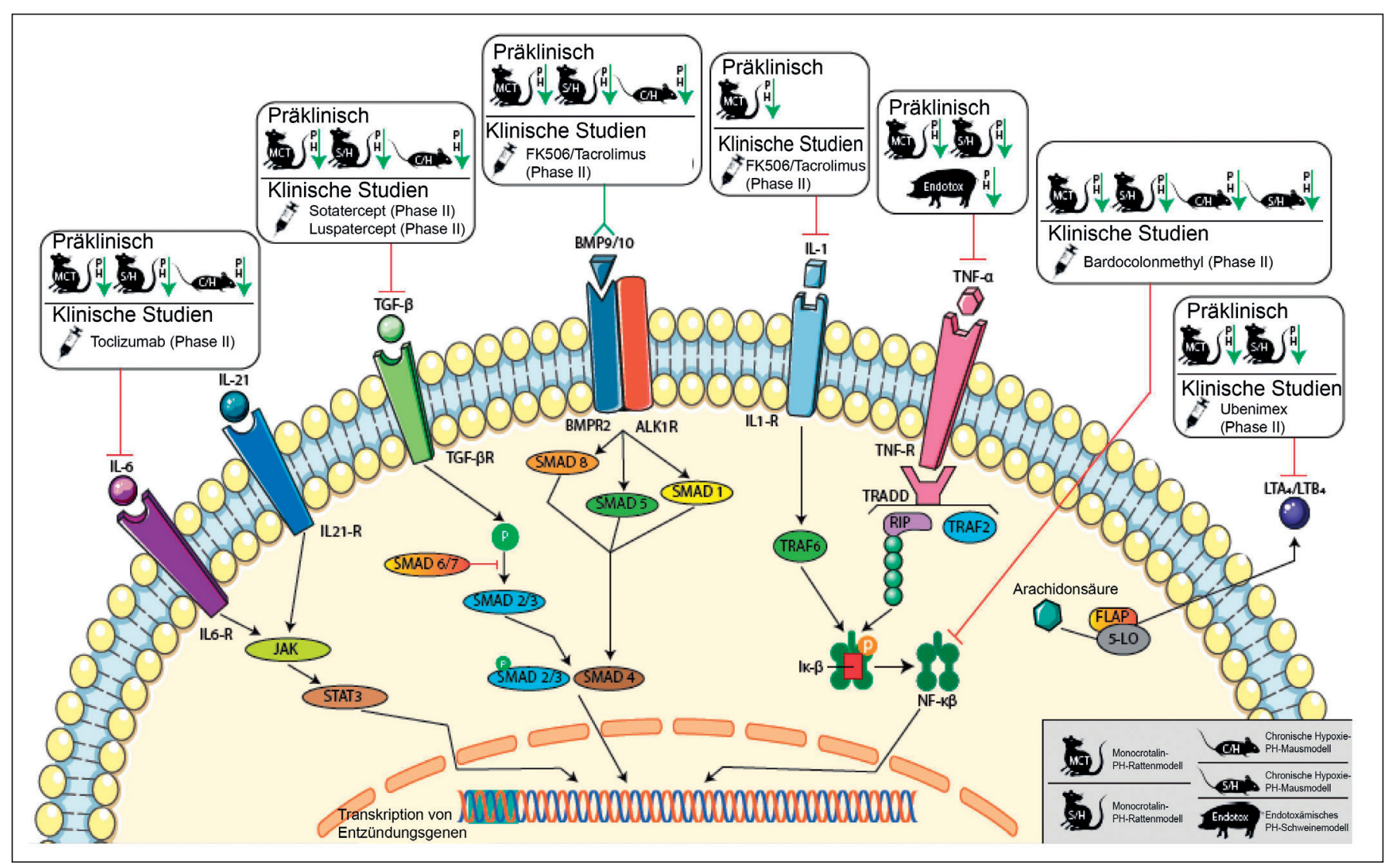

Abb. 2. Zielmoleküle für eine immunmodulatorische Therapie bei PAH. Schematische Übersicht über (prä-)klinische Zielmoleküle für eine immunmodulatorische Therapie bei pulmonale-arterieller Hypertonie (PAH). Eine Erläuterung der Symbole für die präklinischen PH-Tiermodelle findet sich in der Legende in der unteren rechten Ecke. Interleukin (IL); transformierender Wachstumsfaktor beta (TGF- $\beta$ ); Knochenmorphogenetisches Protein (bone morphogentic protein, BMP); Knochenmorphogenetisches Protein-Rezeptor Typ II (BMPR2); Tumornekrosefaktor-a (TNF-a); nuclear factor kappa-light-chain-enhancer of activated B cells (NF-KB,); Leukotrien A4/B4 (LTA4/B4). Januskinasen (JAK); signal transducer and activator of transcription-Proteine (STAT); small mothers against decapentaplegic (SMAD); TNF receptor-associated factor (TRAF); tumor necrosis factor receptor type 1-associated death domain (TRADD); receptor-interacting protein (RIP); Inhibitor von K $\beta$ (IK- $\beta$ ); 5-lipoxygenase activating protein (FLAP); Arachidonsäure 5-Lipoxygenase (5-LO).

dien belegen, dass BMPR2 eine wichtige Rolle im Rahmen der Pathogenese von PAH spielt. Bei der Bindung an knochenmorphogenetische Proteine (BMPs) initiiert BMPR2 intrazelluläre Signalwege, die letztlich zur Hemmung der Proliferation vaskulärer glatter Muskelzellen führen. In glatten Muskelzellen kann die BMP-Signalübertragung direkt durch TGF- $\beta$-Signalwege gehemmt werden, und beteiligte Liganden können im Wettkampf um die Bindung an Typ-II-Rezeptoren als Antagonisten fungieren [123]. BMPR2-Funktionsverlustmutationen sind als Ursache für die Entwicklung von PAH bei Patienten bekannt und führen im Vergleich zu PAH-Patienten ohne BMPR2-Mutation zu einem schwereren Krankheitsverlauf und einem erhöhten Sterberisiko [124]. In einer aktuellen Studie erwies sich BMP9 bei Patienten mit portopulmonaler Hypertonie als sensitiver und spezifischer Biomarker, um ein transplantatfreies Überleben und das Vorliegen einer PAH bei Lebererkrankung zu prognostizieren [125]. In der Lunge von Patienten mit IPAH sowie bei Mäusen mit Hypoxie-induzierter $\mathrm{PH}$ führte die durch eine verminderte BMPR2Expression induzierte Rekrutierung von Makrophagen - unter anderem durch eine gesteigerte Produktion des Chemokins GMCSF (granulocyte macrophage colony-stimulating factor) zu einer Exazerbation der PAH-Symptome [126]. In Nagermodellen, z.B. MCT-Rattenmodell, Su/Hx-PH-Mausmodell und bei Mäusen, denen ein humanes Knock-in-Allel mit BMPR2-Mutation eingeschleust wurde, kann die Aktivierung von BMPR2 ein vaskuläres Remodeling verhindern und den PAH-Phänotyp mit Endothelwachstum und -proliferation abschwächen [127, 128, 129]. Vor Kurzem wurde ein Unternehmen zur Arzneimittelentdeckung (MorphogenIX, Cambridge, UK) gegründet, um BMPs als neuartige Behandlung für PAH zu entwickeln.

Vor dem Hintergrund, dass für die dem BMPR2-Funktionsverlust nachgelagerte Hochregulierung von TGF- $\beta$ eine Korrelation mit der Entwicklung von $\mathrm{PH}$ gezeigt wurde, stellt das Targeting von TGF- $\beta$-Signalwegen möglicherweise ebenfalls einen wirksamen Behandlungsansatz bei PAH dar [130, 131]. In einer aktuellen Studie führte die Behandlung mit TGFBRII-Fc (immunoglobulin-Fc fusion protein TGF- $\beta$ ), einem selektiven, gegen THF-b $1 / 3$ gerichteten TGF- $\beta$-Inhibitor, zur Abschwächung des PH-Phäno- 
typs bei MCT-PH-Ratten sowie bei Ratten und Mäusen mit SU/ Hx-induzierter PH [132]. Derzeit werden so genannte Ligandenfallen, wie etwa Sotatercept und Luspatercept, die eine hohe Selektivität für Mitglieder der TGF- $\beta$-Superfamilie aufweisen, im Rahmen von Phase-II-Studien untersucht (PULSAR-Studie), da sie sich in den vorangegangenen klinischen Phase-I-Studien als erfolgreich erwiesen haben. Diese Ligandenfallen können möglicherweise die BMPR2-Signalübertragung wieder normalisieren und die Gefäßhomöostase wiederherstellen.

Ein weiteres, möglicherweise interessantes Molekül des BMPR2Signalwegs ist der Calcineurin-Inhibitor FK506 (Tacrolimus), der laut Berichten die Expression und Aktivität von BMPR2 erhöht. Bei Ratten mit MCT-PH- und SU/Hx-induzierter PH sowie bei Mäusen mit Hypoxie-induzierter PH revertierte FK506 Berichten zufolge den für einen schweren $\mathrm{PAH}-$ Verlauf verantwortlichen Phänotyp [133]. Bei Patienten mit IPAH führte die Behandlung mit FK506 zu einer Reversion der dysfunktionalen BMPR2-Signalübertragung in den pulmonal-arteriellen Endothelzellen [133]. In einer neueren Phase-IIa-Studie zeigten sich bei der Behandlung von 20 PAH-Patienten mit FK506 eine erhöhte Expression von BMPR2, eine Verbesserung der 6-Minuten-Gehstrecke (6-MWD) sowie bei einigen Patienten eine Verbesserung der serologischen und echokardiografischen Herzinsuffizienz-Parameter. Die Veränderungen waren jedoch nicht signifikant [134]. Nichtsdestotrotz wurde FK506 im Allgemeinen gut vertragen, und diese Studie stützt die Aufnahme einer Phase-IIb-Studie zur Wirksamkeit. Zudem ist Tacrolimus auch gut bekannt für andere immunsupprimierende Wirkungen, etwa die Hemmung von TZellen im Bereich Organtransplantion, so dass die Effekte möglicherweise nicht allein auf den BMPR2-Signalweg beschränkt sind.

$\mathrm{Nf}-\kappa \beta$ (nuclear factor kappa-light-chain-enhancer of activated B cells) ist ein ubiquitärer Transkriptionsfaktor, von dem man weiß, dass er zahlreiche Aspekte der angeborenen und adaptiven Immunfunktionen reguliert. Da er die Expression verschiedener proinflammatorischer Gene für Zytokine und Chemokine induziert, ist NF- $\kappa \beta$ ein wichtiger Regulator für das Überleben, die Proliferation und die Mobilität von Zellen [135]. Das Targeting von NF- $\kappa \beta$ könnte daher einen interessanten neuartigen Therapieansatz für die Behandlung von PAH darstellen. Bei MCT-PHRatten war NF- $\kappa \beta$ nachweislich aktiviert, und durch eine Nf- $\kappa \beta$ blockierende Behandlung konnte der PH-Phänotyp abgeschwächt werden $[136,137,138]$. Diese bessernde Wirkung fand sich ebenfalls bei SU-Hx-Ratten. Hier führte das NF- $\kappa \beta$-Targeting zu stark verminderten Verschlüssen des Lungengefäßlumens [139]. Des Weiteren konnte bei MCT-behandelten transgenen Mäusen mit Überexpression eines herzspezifischen dominant-negativen inhibitorischen Bindungspartners von NF- $\kappa \beta$ (I $\kappa \mathrm{B} \alpha \mathrm{P}$ ) durch die Hemmung von NF- $\kappa \beta$ eine rechtsventrikuläre Hypertrophie $(\mathrm{RVH})$ verhindert werden [140]. Bei Patienten mit PAH war NF$\kappa \beta$ in pulmonalen Lymphozyten, Makrophagen, Endothelzellen und PASMCs nachweislich hoch aktiviert [141].

Derzeit stehen mehrere NF- $\kappa \beta$-Hemmer für eine Beurteilung der Behandlungswirksamkeit bei Patienten mit PH zur Verfügung.
Bardoxolonmethyl ist ein bekannter NF- $\kappa \beta$-Hemmer und zeigt Wirkungen bei der Supprimierung der Aktivierung von Entzündungsmediatoren, der Verstärkung der Bioverfügbarkeit von endothelialem NO, der Besserung von Stoffwechselstörungen, der Supprimierung der Gefäßproliferation sowie der Prävention eines maladaptiven Remodelings [142, 143, 144]. Aktuell wird Bardoxolonmethyl in einer klinischen Phase-II-Studie bei Patienten mit pulmonaler Hypertonie (IPAH, CTD-PAH, PH der WHOGruppe III oder V) beurteilt.

Ein weiterer viel versprechender Kandidat ist Dimethylfumarat (DMF), das aufgrund der Inhibition von NF- $\kappa \beta$ eine starke entzündungshemmende Wirkung besitzt. DMF ist ein Aktivator von NRF2 (transcriptional regulator nuclear factor erythroid 2-related factor 2), das für seine Schlüsselregulierung von Antioxidansgenen bekannt ist [145]. Die NRF2-Funktion ist mit dem NFkB-Signalweg über eine Aktivierung von NRF2 verknüpft, was zu einer Hemmung der NFkB-Signalübertragung und damit zur Induzierung einer entzündungshemmenden Reaktion führt [146]. Berichten zufolge schwächt DMF den PAH-Phänotyp im Mausmodell mit durch chronische Hypoxie und SU/Hx induzierter PH ab [147]. Derzeit werden keine klinischen Studien zur Beurteilung des klinisch-therapeutischen Nutzens von DMF durchgeführt.

Wie oben beschrieben wird durch eine wachsende Zahl an Belegen zunehmend deutlich, dass B-Zellen und Plasmazellen bei der Pathogenese von $\mathrm{PH}$ eine pathologische Bedeutung zukommt. Daher könnte das Targeting von CD20, einem B-Zell-spezifischen Oberflächenmarker, einen viel versprechenden pharmakotherapeutischen Ansatz zur Beurteilung darstellen. Laut einem Fallbericht konnte bei einem jungen Patienten mit SLE eine frühmanifeste PAH durch Behandlung mit Rituximab signifikant gebessert werden [148]. Aktuell führt das National Institute of Allergy and Infectious Diseases (NIAD) eine prospektive, placebokontrollierte, multizentrische, randomisierte Doppelblindstudie der Phase II zur Beurteilung der Wirkung einer Behandlung von Patienten mit SSc-PAH mit Rituximab durch.

Ein weiteres interessantes therapeutisches Zielmolekül ist Leukotrien B4 (LTB4), ein proinflammatorischer Lipidmediator, der durch die sequenziellen Aktivitäten von 5-Lipoxygenase, 5-Lipoxygenase-aktivierendem Protein und Leukotrien-A4-Hydrolase aus Arachidonsäure gebildet wird [149]. Bei MCT-PH-Ratten reduziert der LTB4-Rezeptor-Antagonist (ONO4057) Berichten zufolge nach Behandlung mit MCT eine RVH und verhindert die Entwicklung einer PH [150].

Die unspezifische Inhibition von Leukotrien A4 durch Bestatin (Ubenimex) führte bei Ratten mit MCT-PH und SU/Hx-induzierter PH zur Reversion des PH-Phänotyps [37]. Aktuell wird die Wirksamkeit einer Behandlung mit Bestatin in einer klinischen Phase-II-Studie (LIBERTY-Studie) bei 61 Patienten mit PAH beurteilt. Vorläufige Ergebnisse des Sponsors der Studie (Eiger Biopharmaceuticals, Palo Alto, CA, USA), zeigen allerdings keinen signifikanten Behandlungseffekt im Vergleich zu Placebo. 


\section{Zusammenfassung}

Insgesamt gibt es zunehmende Belege dafür, dass das Immunsystem bei der Pathogenese von PAH und CTEPH eine Schlüsselrolle spielt. In histologischen Untersuchungen sowohl bei PAH als auch bei CTEPH wurde eine extensive Anreicherung von Immunzellen nachgewiesen. Weitere Analysen des Lungengewebes von Patienten mit IPAH und von Lungenbiopsiematerial von Patienten mit CTEPH lieferten überzeugende Belege für die Aktivierung des angeborenen Immunsystems. Die Beteiligung von Makrophagen, Mastzellen und Neutrophilen am pathologischen Geschehen in Form der Produktion proinflammatorischer Zytokine, der Rekrutierung anderer Immunzellen sowie lokaler Entzündung und Schädigung wurde nachgewiesen. In der Lunge von Patienten mit IPAH wurde eine erhöhte Anzahl dendritischer Zellen beobachtet, die über die Antigenpräsentation gegenüber T-Zellen als Bindeglied zwischen dem angeborenen und dem adaptiven Immunsystem fungieren. DCs tragen zur gesteigerten Produktion von Zytokinen und Chemokinen bei und locken so andere Entzündungszellen an den Ort des Geschehens. Bei Patienten mit PAH fand sich eine dysregulierte Th17-Immunität, wodurch eine proinflammatorische Autoimmunumgebung entstand. Darüber hinaus zeigen Patienten mit IPAH vermehrt zirkulierende Autoantikörper, die spezifisch gegen Oberflächenantigen auf Endothelzellen gerichtet sind. Umfassende Forschungsarbeiten zu Biomarkern haben ergeben, dass zahlreiche Entzündungs- und Immunmarker mit den hämodynamischen Parametern und/oder der Prognose bei Pati- enten mit PAH bzw. CTEPH korrelieren. Allerdings sind weitere Untersuchungen erforderlich, um die Eignung dieser Parameter für die klinische Beurteilung von Patienten mit PAH und CTEPH $\mathrm{zu}$ erforschen. Derzeit laufen klinische Studien, mit denen der Nutzen vielversprechender inflammatorischer und immunologischer Zielmoleküle, die in präklinischen Untersuchungen bei PAH ermittelt wurden, beurteilt werden soll. Möglicherweise ist neben den aktuellen Behandlungen, die sich ausschließlich auf eine Vasodilatation stützen, eine Kombination immunmodulatorischer Therapien erforderlich, um eine wirksame Behandlung und Prävention gegen das Fortschreiten dieser Erkrankung zu etablieren.

\section{Disclosure Statement}

Die Autoren erklären, dass keine Interessenskonflikte bestehen.

\section{Lizenzangabe}

Koudstaal T, Boomars KA, Kool M: Pulmonary Arterial Hypertension and Chronic Thromboembolic Pulmonary Hypertension: An Immunological Perspective. J Clin Med. 2020;9(2):E561. (DOI: 10.3390/jcm9020561). ( 2020 by the authors. Licensee MDPI, Basel, Switzerland (Übersetzung; Ergänzende Materialien, Beiträge der einzelnen Autoren, Finanzielle Unterstützung, Danksagungen und Abkürzungen gekürzt), lizensiert unter CC BY 4.0 (https://creativecommons.org/licenses/by/4.0/deed.de).

\section{Literatur}

Die Literatur ist unter www.karger.com/Article/Fulltext/507909 abrufbar. 